\title{
Suppressed Oxygen Inhibition in UV Curable Formulations Using a Diene as an Additive
}

\author{
Haruyuki Okamura $^{1 *}$, Yuuki Nishijima ${ }^{2}$, Daiki Noguchi $^{2}$, Takashi Fukumoto ${ }^{2}$, and Yutaka Suzuki ${ }^{3}$ \\ ${ }^{1}$ Department of Applied Chemistry, Osaka Prefecture University, \\ 1-1, Gakuen-cho, Naka-ku, Sakai, Osaka 599-8531, Japan \\ ${ }^{2}$ Niigata Plant, Kuraray Co., Ltd., \\ 2-28, Kurashiki-cho, Tainai, Niigata 959-2691, Japan \\ ${ }^{3}$ Head Office, Kuraray Co., Ltd., \\ Ote Center Building, 1-1-3, Otemachi, Chiyoda-ku, Tokyo 100-8115, Japan \\ *okamura@chem.osakafu-u.ac.jp
}

\begin{abstract}
We investigated the formulations of acrylates containing a diene as an additive. The mixtures of acrylate monomers, photoinitiators, and an additive were placed in an aluminum pan. The samples were irradiated by a medium pressure mercury lamp without a filter or by a $365-\mathrm{nm}$ light through a band-pass filter. The effect of the initiators, irradiation wavelength, atmosphere, and additive on the conversion of the acryl unit was investigated by photo-DSC measurements. We found that the additive, 1,3-bis[(3-methyl-2-buten-1-yl)oxy]propan-2-ol (DPNG), was the most effective additive for suppressed oxygen inhibition. In addition, the suppressed oxygen inhibition was observed even in thin films of acrylates containing DPNG by in-situ FT-IR measurements.
\end{abstract}

Keywords: Photocuring, Acrylate, Diene, Oxygen inhibition

\section{Introduction}

UV curing materials have been extensively studied due to their wide applicability such as negative-type photoresists, coating materials and printing materials [1]. Especially, UV curing materials formed by a radical mechanism have been widely used due to the wide availability of formulations such as monomers, initiators, etc. However, the radical mechanism influenced by oxygen in air, the so-called "oxygen inhibition", significantly affects the properties of the cured materials. As mentioned in a review article by Liska et al. [2], there are many approaches to overcome the oxygen inhibition. The approaches include controlling the light intensity and wavelength [3,4], using new photoinitiators [5-14], additives such as singlet oxygen generators and scavengers [15-17], hydrogen donors [18,19], reducing agents [19-24], using more reactive monomers [25], and application of a hybrid UV curing system which is a convention of a radical mechanism and other mechanism such as the cationic or addition mechanism [3,26-28].
Theoretical investigations [29-37] are also important in addition to the previously mentioned experimental approaches. We are also interested in this topic, so several approaches for reducing the oxygen inhibition have been reported [4,28]. We have designed and synthesized a novel dimethacrylate monomer having a chalcone moiety. The monomer was applied to the hybrid UV curing system using the photoradical polymerization of methacrylates and photodimerization of chalcone. We found that controlled photodimerization and photoradical polymerization were applicable to the photocuring system with reduced oxygen inhibition [28]. We also found that UV-C LED irradiation was effective to reduce the oxygen inhibition [4].

In this study, we investigated the formulations of acrylates containing novel additives having diene structures. The effect of the structures of additives and initiators, irradiation wavelength, and atmosphere on the conversion of the acryl unit was investigated by photo-DSC [4] and in-situ FT-IR [38] measurements. The photo-DSC technique is a powerful tool regardless of its low reproductivity 
and unsuitable experimental conditions by observing the oxygen inhibition. In addition, insitu FT-IR measurements are straightforward method regardless of the limitation of sample thickness. Reaction mechanism was also discussed in terms of the effect of the light sources applied, the structures of additives, photoinitiators, and acrylates.

\section{Experimental}

\subsection{Materials}

The structures of the chemicals used in this study are shown in Fig. 1. Multifunctional acrylates, i.e., 1,9-nonanediol diacrylate (NDDA) was obtained from Kuraray, and pentaerythritol triacrylate (PETA) was kindly donated by Kuraray and ShinNakamura Chemical Co., Ltd., and were used without further purification. An additive, 1,3-bis[(3methyl-2-buten-1-yl)oxy]propan-2-ol (DPNG) was obtained from Kuraray, and pentaerythritol tetrakis(3-mercaptobutylate) (PEMB) was obtained from Showa Denko K.K. and used as received. Triethanolamine (TEOA) was purchased from Nakarai Tesque and used as received. Photoinitiators, 1-hydroxy-1-cyclohexyl phenyl ketone (HCPK) and diphenyl $(2,4,6-$ trimethylbenzoyl)phosphine oxide (TPO), were obtained from Aldrich and used as received.

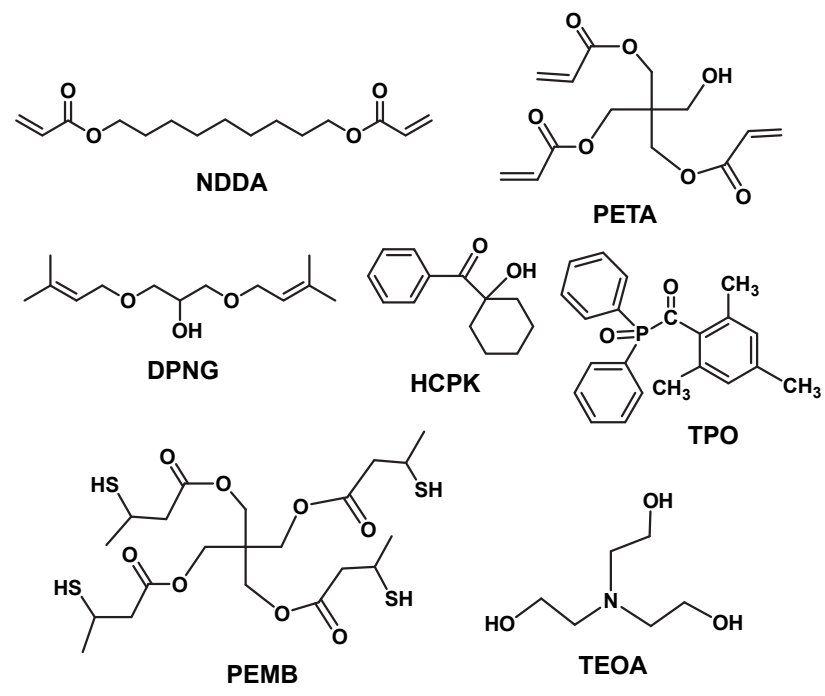

Fig. 1. Structures of chemicals used in this study.

\subsection{Method}

The photo-DSC measurements were carried out as described below. A mixture of multifunctional acrylates, photoinitiators, and additives (2 20 mg) was placed in a 5-mm-diameter aluminum pan. Irradiation was performed using a medium pressure mercury lamp (Hamamatsu LIGHTNINGCURE LC-8, $280 \mathrm{~W}$ ) without filter or a xenon lamp (Asahi Spectra, MAX-301, $300 \mathrm{~W})$ through a band-path filter at $365 \mathrm{~nm}$ combined with a mirror which passes the light below $400 \mathrm{~nm}$ using a Shimadzu UV-DSC system in air or under $\mathrm{N}_{2}$. Conversion of the acryl unit in the blended film was determined using the heat value of $20.6 \mathrm{kcal} / \mathrm{mol}$ for the reaction of the acrylate double bond [39].

The in-situ FT-IR measurements were carried out using a JASCO FT/IR-4200 equipped with a JASCO RS-200 thermal stage and a medium pressure mercury lamp (Hamamatsu LIGHTNINGCURE LC-8, $280 \mathrm{~W}$ ) without filter. The samples were coated or sandwiched with $\mathrm{Si}$ wafer or $\mathrm{CaF}_{2}$ plates to form the films. Conversion of the acryl unit in the film was determined by the in-situ FT-IR measurements using the peak at 1635 $\mathrm{cm}^{-1}$ ascribed to the acryl unit.

\subsection{Measurements}

The UV-vis spectra were obtained using a Shimadzu UV-2400 PC. The intensity of the light was measured by an Orc Light Measure UV-M02 or Ushio USR-45VA. The thickness of the films was determined by a peacock dial thickness gauge (model G, OZAKI Mfg.) or by profilometry (Kosaka Surfcorder ET-3000i).

\section{Results and discussion}

3.1. Characteristics of photoinitiators and light sources

UV curing is generally achieved by photopolymerization which is obtained by the utilization of photoinitiators and photocrosslinking agents. Photoinitiators are the key materials for the UV-curing processes. The first stage of the UV curing system is the absorption of a photon from the incident radiation by the photoinitiators. Light absorption by the photoinitiators requires that the emission line from the light source overlaps with an absorption band of the photoinitiators. Thus, the absorption spectrum of the photoinitiator must be carefully selected [40].

In this study, we selected two compounds as photoinitiators, TPO and HCPK. The photoinitiators are typically type I photoinitiators which are photolyzed to produce two radical species [40]. Figure 2 shows the UV-vis spectra of the photoinitiators in acetonitrile. The molar absorption coefficient values of TPO were 6800 at $254 \mathrm{~nm}$ and 580 at $365 \mathrm{~nm}$, and those of HCPK were 6300 at $254 \mathrm{~nm}$ and 10 at $365 \mathrm{~nm}$ in acetonitrile. 
These values suggest that both photoinitiators absorb 254-nm light to the same extent. On the other hand, HCPK is more transparent than TPO to the 365-nm light.

The selection of light sources is also very important. We investigated the $365-\mathrm{nm}$ light and medium pressure mercury lamp which has multiple line spectra as a conventional light source. The power spectra of the light sources are shown in Fig. 2. TPO absorbs not only $365-\mathrm{nm}$ light but also 405-nm light, 313-nm light and the light below 300 $\mathrm{nm}$ from the medium pressure mercury lamp. The absorption of HCPK is weaker than that of TPO. Thus, the light below $300 \mathrm{~nm}$ strongly affects the photolysis of HCPK.

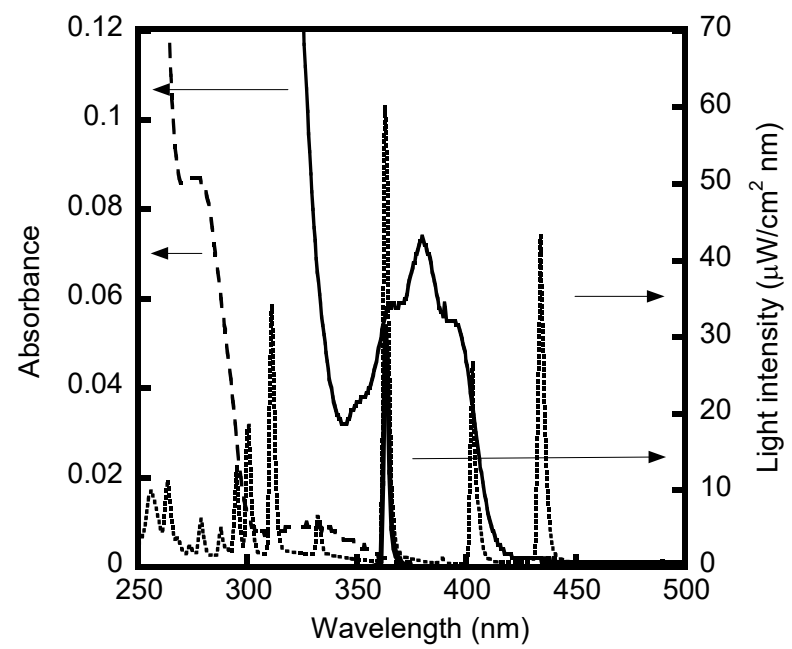

Fig. 2. UV-vis spectra of TPO (solid line) and HCPK (broken line) in acetonitrile $\left(1.0 \times 10^{-4} \mathrm{M}\right)$. Power spectra of medium pressure mercury lamp (dashed line) and 365$\mathrm{nm}$ light (bold line) are also shown.

\subsection{Photocuring behavior}

Photocuring was carried out using the $365-\mathrm{nm}$ light. The mixture of NDDA and HCPK was placed in an aluminum pan. The thickness of the sample was adjusted to 200 and $2000 \mu \mathrm{m}$ to investigate the effect of the sample thickness. The conversion of acrylates in NDDA was calculated using the sum of the heat [38]. The effects of the atmospheric conditions and the sample thickness on the photocuring behavior were investigated (Fig. 3). After irradiation for $10 \mathrm{~min}$, about a $60 \%$ conversion was observed from irradiation under the $\mathrm{N}_{2}$ atmosphere regardless of the DPNG content and thickness of the sample. Upon irradiation in air, decreased conversions were observed for all the samples. The decrease shows the oxygen inhibition under these experimental conditions. The oxygen inhibition clearly observed in the thin sample without DPNG. With the addition of DPNG, the oxygen inhibition was effectively suppressed regardless of the sample thicknesses.

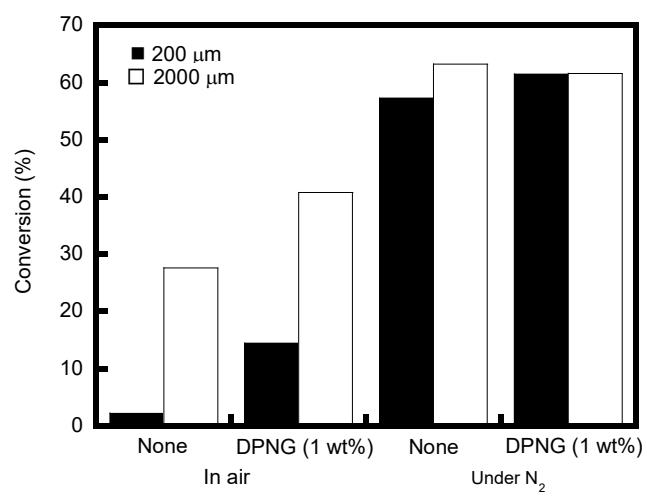

Fig. 3. Conversion of NDDA/HCPK (1:0.01, wt/wt) blended samples with and without DPNG as an additive. Irradiation was carried out at $365 \mathrm{~nm}$ for $10 \mathrm{~min}$. Light intensity: $2.0 \mathrm{~mW} / \mathrm{cm}^{2}$.

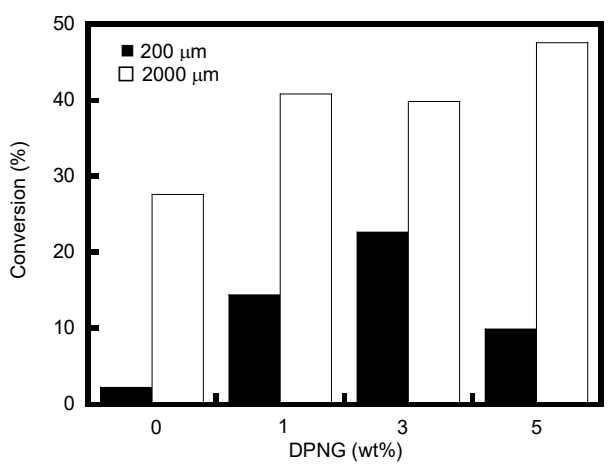

Fig. 4. Effect of DPNG content on conversion of NDDA/HCPK (1:0.01, wt/wt) blended samples. Irradiation was carried out at $365 \mathrm{~nm}$ for $10 \mathrm{~min}$. Light intensity: $2.0 \mathrm{~mW} / \mathrm{cm}^{2}$.

The effect of the DPNG content on the conversion of the NDDA/HCPK $(1: 0.01, \mathrm{wt} / \mathrm{wt})$ blended samples was investigated (Fig. 4). The oxygen inhibition was reduced, reached a minimum value, and increased with the DPNG content for the thin samples. The result suggests that DPNG has both a suppressed oxygen inhibition effect and inhibition of polymerization.

The selection of the light sources is also very important. We investigated the effect of the irradiation wavelength (Fig. 5). The irradiation by a medium pressure mercury lamp showed a slight oxygen inhibition regardless of the DPNG content. The observation may be due to the strong light intensity $\left(12 \mathrm{~mW} / \mathrm{cm}^{2}\right.$ for $\left.365 \mathrm{~nm}\right)$ compared to the $365-\mathrm{nm}$ light $\left(2 \mathrm{~mW} / \mathrm{cm}^{2}\right)$ and has multiple line spectra as shown in Fig. 2. Moreover, the light 
below $300 \mathrm{~nm}$ strongly affects the photolysis of HCPK, which produces an effective polymerization even in air. We reported that the reaction rates of the films upon irradiation below $300 \mathrm{~nm}$ were higher than those upon irradiation at $365 \mathrm{~nm}$. The results clearly showed the effectiveness of the UVC LED lamps on the UV curing in air [31].

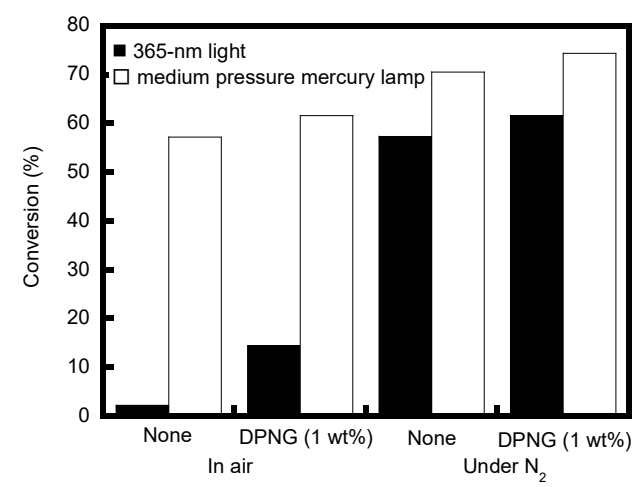

Fig. 5. Effect of light source on conversion of NDDA/HCPK (1:0.01, wt/wt) blended samples. Sample thickness: $200 \mu \mathrm{m}$. Irradiation was carried out for 10 min. Light intensity of $365-\mathrm{nm}$ light: $2.0 \mathrm{~mW} / \mathrm{cm}^{2}$. Light intensity of medium mercury lamp: $12.0 \mathrm{~mW} / \mathrm{cm}^{2}$ at $365 \mathrm{~nm}$.

The effect of photoinitiators on the irradiation was investigated. Figure 6 shows the conversion of NDDA with $1 \mathrm{wt} \%$ photoinitiators upon irradiation. Using TPO as a photoinitiator, a slight oxygen inhibition occurred regardless of the DPNG content and sample thickness. This observation is due to the stronger absorption of TPO at $365 \mathrm{~nm}$, which produced a higher radical concentration sufficient to suppress the oxygen inhibition.

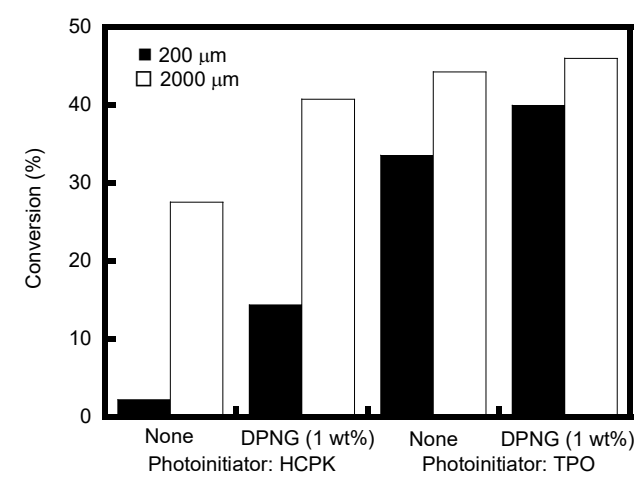

Fig. 6. Effect of photoinitiator on conversion of NDDA/photoinitiator $(1: 0.01, \mathrm{wt} / \mathrm{wt})$ blended samples. Irradiation was carried out at $365 \mathrm{~nm}$ for $10 \mathrm{~min}$. Light intensity: $2.0 \mathrm{~mW} / \mathrm{cm}^{2}$.

The effect of the monomer structure on the conversion of the monomer/HCPK (1:0.01, wt/wt) blended samples was investigated (Fig. 7). Using PETA as a monomer, the oxygen inhibition was slightly observed regardless of the DPNG content and sample thickness. This result suggests that the more viscous PETA suppresses the penetration of oxygen from the surface of the samples, which reduced the oxygen inhibition.

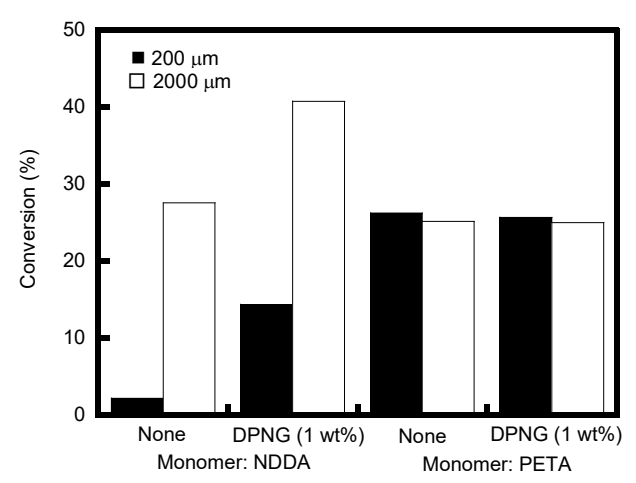

Fig. 7. Effect of monomer on conversion of monomer/HCPK (1:0.01, wt/wt) blended samples. Irradiation was carried out at $365 \mathrm{~nm}$ for $10 \mathrm{~min}$. Light intensity: $2.0 \mathrm{~mW} / \mathrm{cm}^{2}$.

The effect of an additive on the conversion of the NDDA/HCPK (1:0.01, wt/wt) blended samples was investigated (Fig. 8). PEMB is a tetrafunctional thiol which acts as a hydrogen donor. TEOA also acts as a hydrogen donor, which suppresses the oxygen inhibition [2]. The efficiency of the suppressed oxygen inhibition of DPNG is similar to those of PEMB and TEOA. The oxygen inhibition was also suppressed using DPNG and TEOA at the same time. This result suggests that DPNG do not work as a hydrogen donor like TEOA.

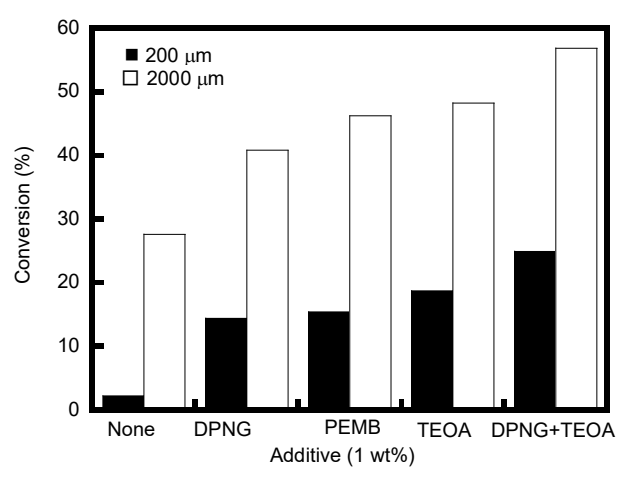

Fig. 8. Effect of additive on conversion of NDDA/HCPK (1:0.01, wt/wt) blended samples. Irradiation was carried out at $365 \mathrm{~nm}$ for $10 \mathrm{~min}$. Light intensity: $2.0 \mathrm{~mW} / \mathrm{cm}^{2}$.

Using thin films $(\sim 5 \mu \mathrm{m})$ of PETA, the effect of DPNG for suppressed oxygen inhibition was clearly 
observed. Figure 9 shows the result of in-situ FTIR measurements of PETA containing HCPK with or without DPNG as an additive. For comparison, a laminated film of DPNG was also measured. The conversions after irradiation for $60 \mathrm{~s}$ were 10 , 56 , and $78 \%$ for the film without DPNG and irradiated in air, the film with $1 \mathrm{wt} \%$ DPNG and irradiated in air, and the laminated film without DPNG, respectively. The result strongly suggests that the addition of DPNG suppress the oxygen inhibition even in thin films.

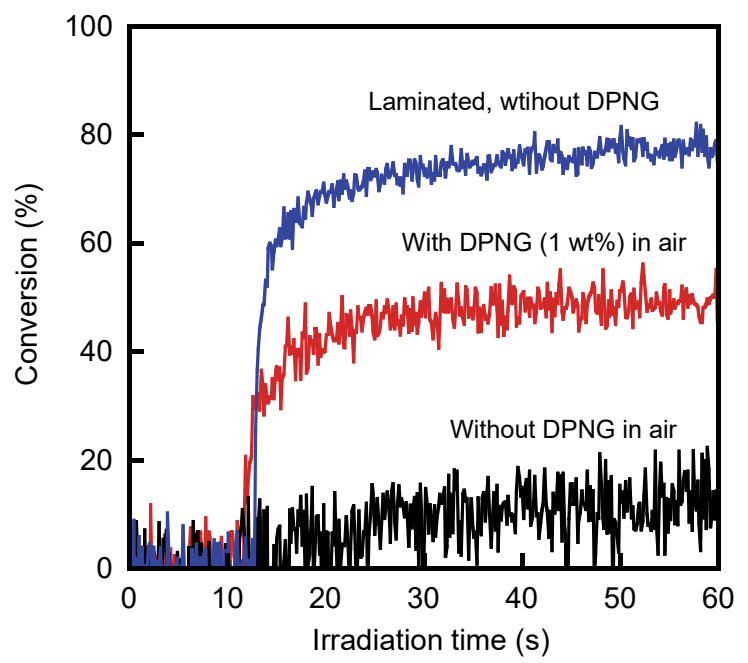

Fig. 9. Conversion of acryl groups of PETA containing 1 wt\% HCPK measured by in-situ FT-IR spectroscopy. Irradiation intensity: $73 \mathrm{~mW} / \mathrm{cm}^{2}$ for $365 \mathrm{~nm}$. Film thickness: $5 \mu \mathrm{m}$.

\subsection{Reaction mechanism}

The plausible reaction mechanism using DPNG as an oxygen scavenger is shown in Scheme 1 . Upon irradiation, a photoinitiator was photolyzed to produce the primary radical. The primary radical reacts with a monomer to produce propagation of the polymerization of the monomers to form a macroradical. Oxygen easily reacts with the macroradical or primary radical to form the peroxy radical, which cannot react again with monomers to form active macroradicals. In the presence of DPNG, the peroxy radical may abstract the active hydrogen in DPNG to produce the DPNG radical. The DPNG radical reacts with oxygen to form the peroxy radical containing the DPNG moiety. The peroxy radical may abstract the active hydrogen in DPNG again to produce the DPNG radical. The regenerated DPNG radical may act as an oxygen scavenger. We consider that the peroxy radical containing the DPNG moiety is unstable and decomposes to form several low-molecular-weight species which do not strongly inhibit the propagation of polymerization. An investigation of the detailed reaction mechanism is now in progress.

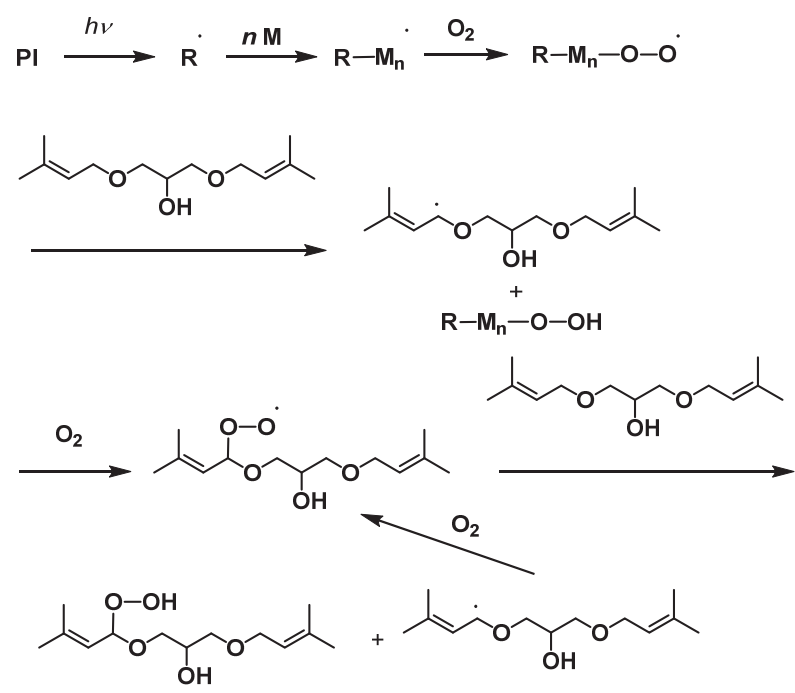

Scheme 1. Plausible reaction mechanism of DPNG as an oxygen scavenger.

\section{Conclusion}

We investigated the formulations of acrylates containing a diene as an additive. The effect of the initiators, irradiation wavelength, atmosphere, and additive on the conversion of the acryl unit was investigated by photo-DSC measurements by irradiation using a medium pressure mercury lamp without a filter or using 365-nm light through a band-pass filter. We found that the DPNG additive was effective additive for suppressed oxygen inhibition. The effect was sensitive to the film thicknesses and the light sources. Content of DPNG strongly affected the conversion of the films due to both a suppressed oxygen inhibition effect and inhibition of polymerization. The suppressed oxygen inhibition was clearly observed using HCPK instead of TPO as a photoinitiator, and NDDA instead of PETA as a monomer. The suppressed oxygen inhibition was also clearly observed in addition of TEOA, a hydrogen donor. The addition of DPNG suppressed the oxygen inhibition even in thin films which was revealed by in-situ FT-IR measurements. We consider that the suppressed oxygen inhibition is due to the regenerated DPNG radical which may act as an oxygen scavenger.

\section{References}

1. J. P. Fouassier and J. F. Rabek, "Radiation 
Curing in Polymer Science and Technology", Elsevier Applied Science, New York (1993).

2. S. C. Ligon, B. Husár, H. Wutzel, R. Holman, and R. Liska, Chem. Rev., 114 (2014) 557.

3. N. L. K. Thiher, S. M. Schissel, and J. L. P. Jessop, Prog. Org. Coat., 138 (2020) 105378.

4. H. Okamura, S. Niizeki, T. Ochi, and A. Matsumoto, J. Photopolym. Sci. Technol., 29 (2016) 99.

5. H. Hou, Y. Gan, J. Yin, and X. Jiang, Adv. Mater. Interfaces, 1 (2014) 1400385.

6. Y. Zhang, Y. He, J. Yan, X. Zhang, R. Bongiovanni, and J. Nie, Polymer, 71 (2015) 93.

7. S. Liang, Y. D. Yang, H. Y. Zhou, Y. Q. Li, and J. X. Wang, Prog. Org. Coat., 114 (2018) 102.

8. H. Salmi, H. Tar, A. Ibrahim, C. Ley, and X. Allonas, Eur. Polym. J., 49 (2013) 2275.

9. J. Yang, C. Xu, W. Liao, Y. Xiong, X. Wang, and H. Tang, Prog. Org. Coat., 138 (2020) 105410.

10. J. Zhou, X. Allonas, A. Ibrahim, and X. Liu, Prog. Polym. Sci., 99 (2019) 101165.

11. J. Yang, C. Xu, Y. Xiong, X. Wang, Y. Xie, Z. Li, and H. Tang, Macromol. Chem. Phys., 219 (2018) 1800256.

12. P. Xiao, F. Dumur, B. Graff, F. Morlet-Savary, L. Vidal, D. Gigmes, J. P. Fouassier, and J. Lalevée, Macromolecules, 47 (2014) 26.

13. J. Zhou, X. Allonas, and X. Liu, Prog. Org. Coat., 120 (2018) 228.

14. J. Zhou, X. Allonas, and X. Liu, J. Photochem. Photobiol. A: Chem., 356 (2018) 580.

15. K. K. Childress, K. Kim, D. J. Glugla, C. B. Musgrave, C. N. Bowman, and J. W. Stansbury, Macromolecules, 52 (2019) 4968.

16. F. Oytun, M. U. Kahveci, and Y. Yagci, J. Polym. Sci. Part A: Polym. Chem., 51 (2013) 1685.

17. N. Rostami, D. Graf, L. Schranzhofer, S. Hild, and T. Hanemann, Prog. Org. Coat., 130 (2019) 221.

18. M. E. Whitely, J. L. Robinson, M. C. Stuebben, H. A. Pearce, M. A. P. McEnery, and E. Cosgriff-Hernandez, ACS Biomater. Sci. Eng., 3 (2017) 409.

19. T. Ping, Q. Wang, Y. Zhou, and J. Nie, J. Photochem. Photobiol. A: Chem., 373 (2019) 171.

20. M. Bouzrati-Zerelli, M. Maier, C. P Fik, C. Dietlin, F. Morlet-Savary, J. P. Fouassier, J. E Klee, and J. Lalevée, Polym. Int., 66 (2017) 504.

21. P. Garra, F. Dumur, F. Morlet-Savary, C. Dietlin,
J. P. Fouassier, and J. Lalevée, Macromolecules, 49 (2016) 6296.

22. B. Wu, X. Chen, Z. Liu, L. Jiang, Y. Yuan, P. Yan, C. Zhou, and J. Lei, Prog. Org. Coat., 124 (2018) 18.

23. P. Garra, A. Kermagoret, A. Al Mousawi, F. Dumur, D. Gigmes, F. Morlet-Savary, C. Dietlin, J. P. Fouassier, and J. Lalevée, Polym. Chem., 8 (2017) 4088.

24. P. Garra, F. Dumur, D. Gigmes, A. Al Mousawi, F. Morlet-Savary, C. Dietlin, J. P. Fouassier, and J. Lalevée, Macromolecules, 50 (2017) 3761.

25. A. Aguirre-Soto, S. Kim, K. Kaastrup, and H. D. Sikes, Polym. Chem., 10 (2019) 913.

26. J. Zhang, M. Frigoli, F. Dumur, P. Xiao, L. Ronchi, B. Graff, F. Morlet-Savary, J. P. Fouassier, D. Gigmes, and J. Lalevée, Macromolecules, 47 (2014) 2811.

27. C. Esposito Corcione, R. Striani, and M. Frigione, Thermochim. Acta, 576 (2014) 47.

28. H. Okamura, Y. Ueda, and M. Shirai, $J$. Photopolym. Sci. Technol., 26 (2013) 245.

29. K. Taki, Y. Watanabe, H. Ito, and M. Ohshima, Macromolecules, 47 (2014) 1906.

30. F. H. Chen, S. Pathreeker, S. Biria, and I. D. Hosein, Macromolecules, 50 (2017) 5767.

31. C. J. Yeh, M. Hu, and K. R. Shull, Macromolecules, 51 (2018) 5511.

32. J. Pierrel, A. Ibrahim, C. Croutxé-Barghorn, and X. Allonas, Polym. Chem., 8 (2017) 4596.

33. F. Courtecuisse, J. Cerezo, C. CroutxéBarghorn, C. Dietlin, and X. Allonas, J. Polym. Chem. Part A: Polym. Chem., 51 (2013) 635.

34. F. Courtecuisse, F. Karasu, X. Allonas, C. Croutxé-Barghorn, and L. van der Ven, Prog. Org. Coat., 92 (2016) 1.

35. J. Christmann, X. Allonas, C. Ley, and C. Croutxé-Barghorn, Polym. Chem., 10 (2019) 1099.

36. P. D. Iedema, V. Schamböck, H. Boonen, M. N. van der Linden, and R. Willemse, Chem. Eng. Sci., 207 (2019) 130.

37. J. Christmann, C. Ley, X. Allonas, A. Ibrahim, and C. Croutxé-Barghorn, Polymer, 160 (2019) 254.

38. H. Okamura and H. Tachi, J. Photopolym. Sci. Technol., 32 (2019) 237.

39. K. S. Anseth, C. M. Wang, and C. N. Bowman, Macromolecules, 27 (1994) 650.

40. H. F. Gruber, Prog. Polym. Sci., 17 (1992) 953. 